\title{
Cascading an Emerging Pattern Based Classifier
}

\author{
Milton García-Borroto ${ }^{1,2}$, José Fco. Martínez-Trinidad ${ }^{2}$, \\ and Jesús Ariel Carrasco-Ochoa ${ }^{2}$ \\ 1 Centro de Bioplantas. Carretera a Moron km 9, Ciego de Avila, Cuba \\ mil@bioplantas.cu \\ 2 Instituto Nacional de Astrofísica, Óptica y Electrónica. Luis Enrique Erro No. 1, \\ Sta. María Tonanzintla, Puebla, México, C.P. 72840 \\ ariel@inaoep.mx, fmartine@inaoep.mx
}

\begin{abstract}
Emerging Pattern classifiers are accurate and easy to understand classifiers. However, they have two characteristics that can degrade their accuracy: global discretization of numerical attributes and high sensitivity to the support threshold value. In this paper, we introduce a novel algorithm to find emerging patterns without global discretization. Additionally, we propose a new method for building cascades of emerging pattern classifiers, which combines the higher accuracy of classifying with higher support thresholds with the lower levels of abstention of classifying with lower thresholds. Experimental results show that our cascade attains higher accuracy than other state-of-the-art classifiers, including one of the most accurate emerging pattern based classifier.
\end{abstract}

Keywords: Classifier Cascades, Understandable classifiers, Emerging pattern classifiers.

\section{Introduction}

The main goal of a supervised classification algorithm is to build a model based on a representative sample of the problem classes [1. This model can be used to predict the class of new objects or to gain understanding of the problem domain.

Classification using Emerging Patterns [2] is a relatively new methodology [3]. An emerging pattern is a combination of attribute values that occurs mostly in a class, which barely appears in the remaining classes; so the presence of a pattern in a query object gives some evidence about the class the object should belong to. After Dong and Li's work, many authors propose algorithms to extract and use emerging patterns for supervised classification [2].

Emerging pattern classifiers are very valuable tools to solve real problems in many fields like Bioinformatics [4], streaming data analysis [5], intruder detection 6] and mining spatio-temporal relationships [7.

The most successful emerging pattern-based classifier family, introduced by Bailey et al. 8] in 2002, makes a global emerging pattern search in the training stage. They make a previous discretization of all numerical features, so resulting patterns use only items with the structure (Feature = value). The discretized objects are ranked using the information of their attribute values, and they are 
represented in a multi-value tree structure, which is a tree-based representation of the complete training sample. Finally, the authors make a depth first traverse of the tree to extract patterns, using a predefined threshold value for the pattern minimal support. These classifiers have two main drawbacks:

- Global discretization on numerical attributes can seriously degrade the classification accuracy.

- High sensitivity to the support threshold value, which makes very hard to find an automated method to estimate it correctly.

In this paper, we introduce a novel algorithm to find emerging patterns, which does not apply global discretization of numerical attributes. It extracts patterns from a collection of decision trees, using a specialized procedure. To find a representative collection of patterns, it uses a novel object weighting scheme. This algorithm applies local discretization, using only the attribute values appearing in the current node objects.

It is important to highlight that we are not introducing a new method for inducing decision trees or forests. Our algorithm builds decision trees to extract the emerging patterns from them, but the induced trees are completely discarded after the extraction procedure.

Additionally, we propose a new method for building cascades of emerging pattern based classifiers. The method starts estimating the minimal support used in the first classifier and the maximum abstention allowed to the last classifier. Then, it creates a cascade of classifiers using decreasing support values, from the maximum inferred value to the value that attains the maximum allowed abstention. This method obtains an ensemble that classifies unseen objects using emerging patterns with the highest possible support.

The paper is organized as follows: section 2 presents a brief revision about classification using emerging patterns, section 3 introduces the new algorithm for mining emerging patterns without global discretization, section 4 presents the method to build a cascade of emerging patterns based classifiers, section 5 shows the experimental results, and section 6 presents the conclusions.

\section{Classification Using Emerging Patterns}

A pattern is an expression, defined in a language, which describes a collection of objects; the objects described by a pattern are named the pattern support. In a supervised classification problem, we say that a pattern is emerging if its support increases significantly from one class to the others [3]. Emerging patterns are usually expressed as combinations of feature values, like (Color $=$ green, Sex $=$ male, Age $=23)$ or as logical properties, like $[$ Color $=$ green $] \wedge[$ Sex $=$ male $] \wedge$ [Age $>23]$.

Most algorithms for emerging pattern mining have as goal to find the patterns that satisfy a desired property: being supported by a single class (JEP), minimality over subset inclusion (SJEP), or tolerance on noisy objects (NEP) 9. These algorithms have the following steps: 
1. Selection of the minimal support threshold $\mu$

2. Global discretization of numerical attributes

3. Representation of the transformed objects using a particular structure

4. Traversing the structure to find emerging patterns

5. Pattern filtering

Using this traditional algorithm might have two important drawbacks:

1. Global discretization of numerical attributes could drastically degrade the classifier accuracy, since an emerging pattern relates a combination of feature values with a class. Therefore, discretizing a numerical attribute without considering the values of other features could hide important relations.

In Table 1, we can see that SJEPC [9], one of the most accurate classifiers of the family, obtains very poor accuracies in databases like Iris, while all other classifiers attain accuracies above 93\%. In some other databases, SJEPC is unable to extract even a pattern, because most numerical features are discretized into a single categorical value.

2. High sensitivity to the support threshold value. The accuracy of the classifier can have serious accuracy degradation on small variations of the minimal support value. For example, in chess and census databases, the accuracy drops $3 \%$ with a variation of 2 in the threshold 8 .

Emerging Pattern classifiers are not frequently used in ensembles, because they are complex and stable classifiers. Nevertheless, they have been used as base classifiers in ensembles using Bagging and Boosting [10 methods. The Boosted [11] and the Bagged [12] Emerging Pattern classifiers are more accurate than the base classifier, but they do not solve the mentioned drawbacks.

\section{Crisp Emerging Pattern Mining (CEPM)}

In this section, we introduce CEPM, a new emerging pattern mining algorithm with local discretization of numerical features. It extracts patterns from a collection of C4.5 decision trees [13], using an special pattern mining procedure. To guarantee that CEPM finds a representative collection of patterns, it uses a novel object weighting scheme.

The tree induction procedure (InduceTree, steps 2 and 16, Algorithm 2) has the following characteristics:

- Candidate splits are binary. Nominal attributes use properties like $[$ Feature $=a]$ and $[$ Feature $\neq a]$ for each of its values; numerical attributes use properties like $[$ Feature $>n]$ and $[$ Feature $\leq n]$ for all candidate cut points.

- If a node has less than $\mu$ objects, it is not further split because it cannot generate emerging patterns.

- To select the best split, the algorithm evaluates the weighted information gain. The weighted information gain is a modification of the information gain to use weighted probabilities for each class and child node (Equation 1) Note 
Data: $T$ - training sample, maxIter - maximum number of iterations, $\mu$ minimum support ratio

Result: EPS - Mined Patterns, abstentionRatio - Abstention ratio of EPS with respect to $T$

1 forall $o \in T$ do $w_{o} \leftarrow 1$;

/* Simplify procedure deletes duplicated and non-minimal patterns */

2 EPS $\leftarrow$ Simplify (InduceTree $(T, w, \mu))$;

3 averageSupport $\leftarrow \frac{\sum_{e p \in E P S} \operatorname{support}(e p)}{|E P S|}$;

$4 i \leftarrow 0$;

5 repeat

6 AbstentionCount $\leftarrow 0$;

// Weight recalculation

$7 \quad$ foreach $o \in T$ do

$8 \quad E P c \leftarrow\{$ Patterns that support $o$, belonging to its class $\}$;

$9 \quad E P n c \leftarrow\{$ Patterns that support $o$, belonging to a different class $\}$;

$10 \quad$ support $=\sum_{e p \in E P n c} \sup (e p)-\sum_{e p \in E P c} \sup (e p)$;

$11 \quad w_{o}=\operatorname{arccot}\left(\right.$ DesiredSupport $\left.\cdot \frac{\text { support }}{\text { averageSupport }}\right) / \pi$;

12 if any pattern support o then

AbstentionCount $\leftarrow$ AbstentionCount +1

end

EPS $\leftarrow$ Simplify $($ EPS InduceTree $(T, w, \mu)) ;$ $i \leftarrow i+1$

18 until $i=$ maxIter $O R$ no new pattern was added in this iteration;

Algorithm 1. Pseudocode of the algorithm CEPM

that objects with weight close to 0 have low influence in the determination of the best split.

$$
P_{\text {Class }}=\frac{\sum_{o \in \text { Class }} w_{o}}{\sum w_{o}}, \quad P_{\text {child }}=\frac{\sum_{o \in \text { child }} w_{o}}{\sum w_{o}}
$$

During the tree induction, every child node having at least $\mu$ objects in a class, and at most one object in the complement of that class, generates a new emerging pattern. This pattern consists in the conjunction of the properties from the node to the root. For example, from the decision tree in Figure 1 we can extract the patterns $($ Length $>30) \wedge($ Color $=$ red $)$ from class Good, and the pattern $($ Length $\leq 30) \wedge($ Fly $=$ false $)$ from class Bad.

Additionally, CEPM extracts patterns while evaluating the splits, even if a split has not the optimal gain. Any child node having at least $\mu$ objects in a class, and at most one object in the complement of that class, generates an emerging pattern. For example, Figure 2 shows two candidate splits, using different properties. Although the first one has a higher information gain, the second contains the emerging pattern $(E d a d<20)$. So, the pattern is extracted even if the split is discarded.

CEPM iteratively induces different decision trees, updating the object weights after each iteration. The algorithm updates weights using Equation 2. 


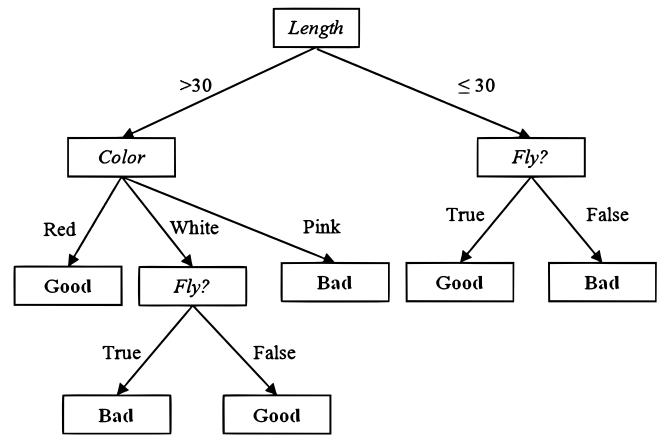

Fig. 1. Example of decision tree with three attributes and two classes

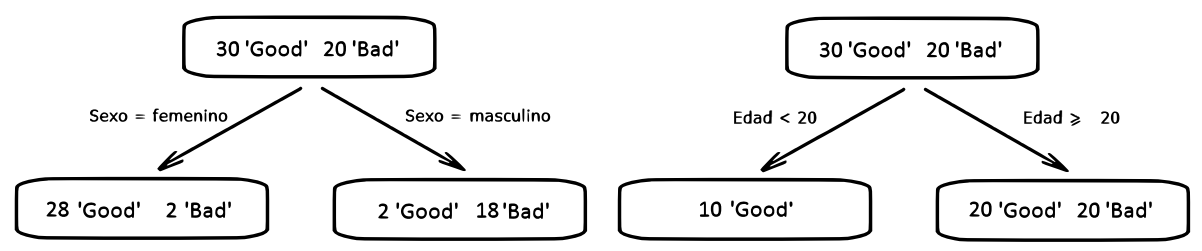

Fig. 2. Example of an emerging pattern in a non-optimal candidate split

$$
w_{o}=\frac{\operatorname{arccot}\left(\text { DesiredSupport } \cdot \frac{\text { Support }_{o}}{\text { averageSupport }}\right)}{\pi}
$$

where

- Support $t_{o}$ is the sum of the support of the patterns contained in $o$. If the pattern belongs to a different class than $o$, its support is multiplied by -1

- averageSupport is the average support of the patterns found in the first built tree

- arccot is the inverse cotangent function

Equation 2 values ranges from 0 to 1 , because arccot ranges between 0 and $\pi$ Figure 3. An object obtains a weight close to 1 if it has a negative total support lower than 5 . In this case, it is necessary to mine more patterns to support the object to its own class. On the contrary, a weight close to 0 means the object has total support above 5 . In this case, no more patterns are necessary for this object, and it is virtually ignored in gain calculations. As we can see in Figure 3. value 5 and -5 are both distinctive in the arccot function, because they are the points where the function values starts to be close to the asymptotes. That is why we use value 5 in the equation 2 ,

The pseudocode of CEPM appears in Algorithm[1 It is worth to mention that CEPM returns a set of minimal emerging patterns with support greater or equal to $\mu$. Pattern minimality is considered with respect to the subset inclusion of 


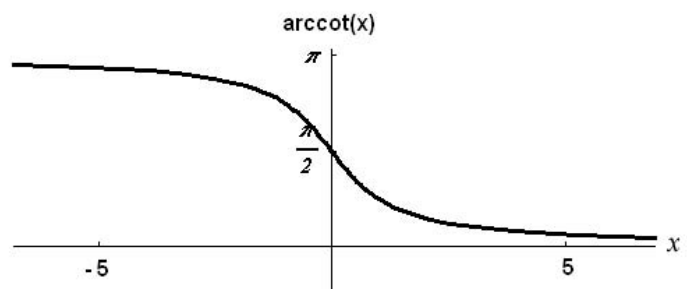

Fig. 3. The arccot function

the pattern component properties. Additionally, it returns the abstention ratio, which is the ratio of objects that are not covered by the resultant patterns.

\section{Cascading CEPM-Based Classifiers}

Data: $T$ - training sample

Result: Classifiers - Cascade of classifiers. The first classifier is the one built with the highest $\mu$ value

1 (MaxSupport, MaxAbstRate) $\leftarrow$ InferParams $(T) / /$ in Algorithm 3

2 currentSupport $\leftarrow$ MaxSupport;

3 step $\leftarrow \max \left\{\frac{\text { current Support }}{10}, 1\right\}$;

4 while currentSupport $>1$ AND currentAbstention $>$ MaxAbstRate do

$5 \mid$ (patterns, currentAbstention $) \leftarrow C E P M(T$, maxIter $=120, \mu=$ currentSupport) ;

if currentAbstention $<0.5$ then

$7 \quad \mid$ Add a new classifier using patterns to Classifiers

$8 \quad$ currentSupport $\leftarrow$ currentSupport - step ;

9 if currentSupport $=0$ AND abstentionRatio $>=$ Max AbstRate then

$10 \quad$ currentSupport $\leftarrow$ step -1 ;

$11 \mid$ step $\leftarrow \max \left\{\frac{\text { currentSupport }}{10}, 1\right\}$;

13 end

Algorithm 2. Pseudocode of the algorithm CascadeCEPM

A cascade classifier is a type of ensemble where a single classifier is active at each time [10]. To classify a query object the first classifier is activated and returns the class. If a classifier is not sure enough about the correct classification, it passes the query object to the next classifier in the chain. Cascading classifiers approach is better than multi-expert methods when the topmost classifiers can handle most objects with higher accuracy, but they are unable to classify some other objects [14].

Emerging pattern classifiers with different minimal support $\mu$ are good candidates for cascading; a classifier using patterns with higher $\mu$ values, is more accurate but could reject to classify more objects. Then, to build a cascade of 
Data: $T$ - training sample

Result: MaxSup - Higher $\mu$ used in the classifier ensemble, AbstentionRate Maximum abstention rate allowed to the classifier with lowest $\mu$ in the ensemble

1 (Patterns, AbstentionRate $) \leftarrow C E P M(T, \max I t e r=20, \mu=2)$;

/* Using the highest support value makes MaxSupport to be high enough; checking that at least half of the objects are supported makes MaxSupport to be not too high

$2 \operatorname{Max} S u p \leftarrow$ Highest support value such that at least half of the objects in $T$ are supported for at least a pattern in Patterns ;

3 return (MaxSup, AbstentionRate)

\section{Algorithm 3. Pseudocode of the algorithm InferParams}

emerging pattern classifiers, the first classifier should be built with a high $\mu$ value. The remaining classifiers in the cascade should have a $\mu$ value lower than the $\mu$ value of their predecessors, for allowing them to classify uncovered objects.

Our novel cascading creation method, named CascadeCEPM, appears in Algorithm 2. It starts inferring the support of the topmost classifier (MaxSupport) and the maximal abstention rate allowed for the lower classifier (MaxAbstRate). CascadeCEPM creates classifiers starting with $\mu=$ MaxSupport, decrementing $\mu$ for each new classifier until it finds an abstention rate lower than max AbstRate or $\mu=1$. For decrementing $\mu$, CascadeCEPM uses a calculated Step (see Algorithm 2), because if MaxSupport is high, decrementing $\mu$ by 1 might be too costly.

Some important remarks:

1. MaxSupport is inferred based on two criteria. If it is higher than the optimum, the algorithm makes costly unnecessary iterations; otherwise, if it is lower than the optimum, better models (with higher $\mu$ ) are disregarded.

2. The value 120 (Algorithm 2, Step 5) is the maximum number of iterations of the algorithm CEPM. It was introduced because, in some databases, CEPM has an slow convergence. We determine this value experimentally; using higher values does not alter the classifier accuracy.

3. MaxAbstRate is inferred using $\mu=2$, so it measures the maximum expected abstention of a pattern based classifier. A maximum iteration value equal to 20 , instead of 120 , speeds up the procedure (Algorithm 3, step 1).

4. We dismiss classifiers with abstention level higher than 0.5, because they were inaccurate in most of the tested databases.

5. The condition currentAbstention $<0.5$ in Algorithm 2 (step 6) discards inaccurate classifiers, having high abstention levels.

CascadeCEPM creates a cascade of emerging pattern classifiers, each one using as the decision rule the highest sum of support. Given a query object, the first classifier returns the most supported class; if no pattern supports the object or there is a tie, the classifier refuses to classify and activates the next classifier in the cascade. If the last classifier cannot classify the query object, the whole cascade refuses to return a classification. 


\section{Experimental Results}

To compare the performance of CascadeCEPM, we carried out some experiments over 18 well-known databases from the UCI Repository of Machine Learning [15]. We selected five state-of-the-art classifiers: 3 Nearest Neighbors [16], Bagging and Boosting [10], Random Forest [17, C4.5 [13. For each classifier, we used the Weka 3.6.1 implementation [18] with its default parameters. We also tested the behavior of the emerging pattern based classifier SJEPC [9, with the minimal support threshold suggested by their authors.

Table 1. Accuracy results of compared classifiers. The highest accuracy per database is bolded

\begin{tabular}{l|ccccccc}
\hline DBName & 3NN & Boost & Bagg & C4.5 & RandFor & SJEPC CascCEPM \\
\hline balance-scale & $\mathbf{8 5 . 4 4}$ & 71.70 & 82.58 & 77.62 & 79.37 & 16.02 & 82.73 \\
breast-cancer & 70.31 & 72.40 & 70.96 & $\mathbf{7 3 . 4 4}$ & 65.75 & 44.47 & 72.34 \\
cleveland & 82.51 & $\mathbf{8 4 . 1 5}$ & 79.88 & 78.19 & 78.57 & 77.90 & 81.51 \\
haberman & 70.56 & 70.90 & $\mathbf{7 2 . 4 7}$ & 67.97 & 67.61 & 0.00 & 71.56 \\
hayes-roth & 71.43 & 53.57 & 75.00 & $\mathbf{8 9 . 2 9}$ & 85.71 & 0.00 & 75.00 \\
heart-c & 81.18 & $\mathbf{8 3 . 1 8}$ & 81.85 & 76.23 & 80.89 & 78.60 & 82.18 \\
heart-statlog & 79.26 & $\mathbf{8 0 . 7 4}$ & 79.26 & 79.26 & 79.26 & 64.81 & 80.00 \\
hepatitis & 81.96 & 81.21 & $\mathbf{8 2 . 0 0}$ & 78.75 & 81.33 & 77.46 & 81.33 \\
iris & 96.00 & $\mathbf{9 6 . 6 7}$ & 93.33 & 94.00 & 94.67 & 66.67 & 95.33 \\
liver-disorders & 65.47 & 66.08 & 68.68 & 68.70 & $\mathbf{7 0 . 7 4}$ & 0.00 & 69.89 \\
lymph & $\mathbf{8 5 . 9 0}$ & 75.67 & 77.67 & 78.48 & 79.86 & 51.48 & 82.52 \\
monks-problem-1 & 50.00 & 50.00 & 50.00 & 50.00 & 50.00 & 57.87 & $\mathbf{1 0 0 . 0 0}$ \\
monks-problem-2 & 51.39 & 50.00 & 55.09 & 59.72 & 58.56 & 34.03 & $\mathbf{7 9 . 1 7}$ \\
monks-problem-3 & 50.00 & 50.00 & 50.00 & 50.00 & 50.00 & 63.66 & $\mathbf{9 7 . 4 5}$ \\
spect & 64.71 & 66.84 & 61.50 & 66.84 & 62.03 & 0.00 & $\mathbf{8 3 . 4 2}$ \\
tic-tac-toe & $\mathbf{9 8 . 5 4}$ & 73.49 & 91.02 & 83.82 & 91.86 & 91.34 & 94.36 \\
vote & 91.97 & 94.72 & 95.18 & $\mathbf{9 6 . 1 0}$ & $\mathbf{9 6 . 1 0}$ & 91.08 & 94.49 \\
wine & 96.05 & 87.48 & 94.31 & 92.65 & $\mathbf{9 7 . 1 6}$ & 55.07 & 94.97 \\
\hline
\end{tabular}

We performed 10-fold cross validation, averaging the results. In both SJEPC and CascadeCEPM we reported the abstentions as errors. In these objects, the classifier is unable to assign a class; returning the majority or a random class could hide these undesirable cases. In Table 1, we can find the accuracy results, in percent.

Experimental results show that SJEPC has low accuracy values in many databases, compared with other classifiers. In those databases, most numerical attributes were transformed in a single categorical attribute, and therefore they were discarded.

In order to determine if the differences in accuracy are statistically significant, we performed a pairwise comparison between our classifier and the others. Each cell in Table 2 contains the number of databases where our classifier Win/Lose/Tie to each other classifier. We detect ties using a two-tailed T-Test 
Table 2. Pairwise comparison (Win/Loss/Tie) between our classifier and the others

\begin{tabular}{l|cccccc}
\hline & $3 \mathrm{NN}$ & AdaBoost Bagging & C4.5 & RandFor SJEPC \\
\hline cascade & $8 / 3 / 7$ & $10 / 1 / 7$ & $6 / 0 / 12$ & $12 / 1 / 5$ & $10 / 2 / 6$ & $18 / 0 / 0$ \\
\hline
\end{tabular}

[19] with significance of 0.05 . The pairwise comparison shows that, in the tested databases, CascadeCEPM is more accurate than any other classifier, using the selected databases.

Like previous emerging pattern classifier, CascadeCEPM scales better to adding new objects than to adding new features.

\section{Conclusions}

In this paper, we introduced CEPM, a new algorithm for mining Emerging Patterns using local discretization of numerical values. Our algorithm solves the main problem of algorithms using global discretization, which makes them useless in some databases. CEPM extracts patterns from a collection of decision trees, using a specialized extraction procedure. For obtaining a collection of representative patterns, CEPM uses a novel object weighting scheme.

Additionally, this paper proposes CascadeCEPM, a new cascading method for Emerging Pattern classifiers. CascadeCEPM infers the maximal and minimal thresholds, generating a cascade of classifiers using selected threshold values in between. Experimental results show that CascadeCEPM is a more accurate method than SJEPC in most databases. A pairwise comparison reveals that it is also more accurate than other state of the art classifiers.

In the future, we will work on speeding up the algorithm to estimate the support thresholds of the classifiers in the ensemble.

\section{Acknowledgments}

This work is partly supported by the National Council of Science and Technology of México under the project CB-2008-01-106443 and grant 25275.

\section{References}

1. Berzal, F., Cubero, J.-C., Sánchez, D., Serrano, J.M.: Art: A hybrid classification model. Machine Learning 54, 67-92 (2004)

2. Ramamohanarao, K., Fan, H.: Patterns based classifiers. World Wide Web 10(1), 71-83 (2007)

3. Dong, G., Li, J.: Efficient mining of emerging patterns: Discovering trends and differences. In: Proceedings of the Fifth ACM SIGKDD International Conference on Knowledge Discovery and Data Mining, San Diego, California, United States, pp. 43-52. ACM, New York (1999)

4. Quackenbush, J.: Computational approaches to analysis of dna microarray data. In: IMIA Yearbook of Medical Informatics, pp. 91-103 (2006) 
5. Alhammady, H.: Mining streaming emerging patterns from streaming data. In: IEEE/ACS International Conference on Computer Systems and Applications (Amman), pp. 432-436 (2007)

6. Chen, L., Dong, G.: Masquerader detection using oclep: One-class classification using length statistics of emerging patterns. In: WAIMW '06: Proceedings of the Seventh International Conference on Web-Age Information Management Workshops, Washington, DC, USA, p. 5. IEEE Computer Society, Los Alamitos (2006)

7. Celik, M., Shekhar, S., Rogers, J.P., Shine, J.A.: Sustained emerging spatiotemporal co-occurrence pattern mining: A summary of results. In: ICTAI '06: Proceedings of the 18th IEEE International Conference on Tools with Artificial Intelligence, Washington, DC, USA, pp. 106-115. IEEE Computer Society, Los Alamitos (2006)

8. Bailey, J., Manoukian, T., Ramamohanarao, K.: Fast algorithms for mining emerging patterns. In: Elomaa, T., Mannila, H., Toivonen, H. (eds.) PKDD 2002. LNCS (LNAI), vol. 2431, pp. 187-208. Springer, Heidelberg (2002)

9. Fan, H., Ramamohanarao, K.: Fast discovery and the generalization of strong jumping emerging patterns for building compact and accurate classifiers. IEEE Transactions on Knowledge and Data Engineering 18(6), 721-737 (2006)

10. Kuncheva, L.I.: Combining Pattern Classifiers. Methods and Algorithms. Wiley Interscience, Hoboken (2004)

11. Sun, Y.: Boosting an associative classifier. IEEE Trans. on Knowl. and Data Eng. 18(7), 988-992 (2006); Member-Wang, Y. and Fellow-Wong, A.K.C

12. Fan, H., Fan, M., Ramamohanarao, K., Liu, M.: Further improving emerging pattern based classifiers via bagging. In: Ng, W.-K., Kitsuregawa, M., Li, J., Chang, K. (eds.) PAKDD 2006. LNCS (LNAI), vol. 3918, pp. 91-96. Springer, Heidelberg (2006)

13. Quinlan, J.R.: C4.5: Programs for Machine Learning. Morgan Kaufmann Publishers Inc., San Francisco (1993)

14. Alpaydin, E., Kaynak, C.: Cascading classifiers. Kybernetica 34(4), 369-374 (1998)

15. Merz, C., Murphy, P.: Uci repository of machine learning databases. Technical Report, University of California at Irvine, Department of Information and Computer Science (1998)

16. Dasarathy, B.D.: Nearest Neighbor (NN) Norms: NN Pattern Classification Techniques. IEEE Computer Society Press, Los Alamitos (1991)

17. Ho, T.K.: The random subspace method for constructing decision forests. IEEE Transactions on Pattern Analysis and Machine Intelligence 20(8), 832-844 (1998)

18. Frank, E., Hall, M.A., Holmes, G., Kirkby, R., Pfahringer, B., Witten, I.H.: 'Weka: A machine learning workbench for data mining. In: Maimon, O., Rokach, L. (eds.) Data Mining and Knowledge Discovery Handbook: A Complete Guide for Practitioners and Researchers, pp. 1305-1314. Springer, Berlin (2005)

19. Dietterich, T.G.: Approximate statistical tests for comparing supervised classification learning algorithms. Neural Computation 10(7), 1895-1923 (1998) 